\title{
Abundância, Composição e Diversidade de Arctiinae (Lepidoptera, Arctiidae) em um fragmento de floresta na Amazônia Oriental em Altamira, PA, Brasil
}

\author{
José Augusto TESTON¹ㄹ Jessé Bucioli NOVAES², José Otávio Barros ALMEIDA JÚNIOR³
}

\begin{abstract}
RESUMO
Este estudo avaliou a fauna de Arctiinae em um fragmento de floresta primária em Altamira, Pará, na Amazônia Oriental brasileira. As mariposas foram amostradas durante dois anos (de agosto de 2007 a julho de 2009), com auxílio de armadilha luminosa. Foram medidos os seguintes parâmetros: riqueza, abundância, constância, índices de diversidade e uniformidade de Shannon (H' e E') e de Brillouin (H e E) e o índice de dominância de Berger-Parker (BP). As estimativas de riqueza, foram efetuadas através dos procedimentos não paramétricos, "Bootstrap", "Chao 1", "Chao 2", "Jackknife 1", "Jackknife2" e "Michaelis-Mentem". Foram capturados 466 exemplares pertencentes a 78 espécies de Arctiinae, das quais 12 são novos registros para o Estado. Os valores dos parâmetros analisados para todo o período foram: $H^{\prime}=3,08, E$ ' $=0,708, H=2,86, E=$ 0,705 e $\mathrm{BP}=0,294$. As comunidades dos meses menos chuvosos foram mais diversas. Os estimadores previram o encontro de 17 a 253 espécies a mais.
\end{abstract}

PALAVRAS-CHAVE: Biodiversidade, Mariposas, Noctuoidea, Região Neotropical

\section{Abundance, Composition and Diversity of Arctiinae (Lepidoptera, Arctiidae) in a forest fragment in the Eastern Amazon in Altamira, PA, Brazil}

\section{ABSTRACT}

This study evaluated the Arctiinae fauna in a fragment of primary forest in Altamira, Pará, Eastern Amazonia, Brazil. The moths were sampled during two years (from August 2007 to July 2009) with a light trap. The following parameters were measured: richness, abundance, constancy, diversity and evenness indices (Shannon H 'and E') and Brillouin (H and E), and dominance index of Berger-Parker (BP). The richness estimatives were made by nonparametric procedures, Bootstrap, Chao 1, Chao 2, Jackknife 1, Jackknife2 and Michaelis-Mentem. Four hundred and sixty-six individuals of 78 species of Arctiinae were captured, of which twelve are new records for Pará State. Values of parameters analyzed for the entire period were: $\mathrm{H}^{~}=$ $3.08, E^{\prime}=0.708, H=2.86, E=0.705$, and $\mathrm{Sp}=0.294$. Communities of the less rainy months were more diverse. Estimators predicted an increase between 17 to 253 in species richness.

KEYWORDS: Biodiversity, Moths, Neotropical Region, Noctuoidea

${ }^{1}$ Universidade Federal do Oeste do Pará - UFOPA, Programa de Pós-Graduação em Recursos Naturais da Amazônia (PGRNA) e Programa de Ciências Biológicas, Av. Marechal Rondon, s/n - Bairro Caranazal - 68040-070 - Santarém, PA. E-mail: jateston@ufpa.br

2 Universidade Federal do Pará - UFPA, Faculdade de Ciências Biológicas, Rua Coronel José Porfírio, 2515 - Bairro São Sebastião - 68372-040 - Altamira, PA. E-mail: jessebnovaes@ yahoo.com.br

3 Universidade Federal do Pará - UFPA, Faculdade de Ciências Biológicas, Rua Coronel José Porfírio, 2515 - Bairro São Sebastião - 68372-040 - Altamira, PA. E-mail: otavioalmeida18@ hotmail.com 


\section{INTRODUÇÃO}

Nas florestas tropicais encontramos a maior diversidade de espécies, apesar de ocuparem apenas $7 \%$ de extensáo do planeta (Primack e Rodrigues 2001). Entre essas, destaca-se a floresta amazônica, que abriga pelo menos 40.000 espécies de plantas, 427 de mamíferos, 1.294 de aves, 378 de répteis, 427 de anfíbios, 3.000 de peixes, 3.000 de abelhas, 500 de aranhas, 1.800 de borboletas, 200 de diplópodes, 3.000 de formigas, 220 de marimbondos e 100 de minhocas (Overal 2001; Silva e Garda 2011).

Atualmente, os perigos para a biodiversidade das suas florestas na Amazônia abrangem: a exploração madeireira, o desmatamento, as queimadas, a fragmentação, a mineração, a extinção da fauna, a invasão de espécies exóticas, o tráfico de animais silvestres e as mudanças climáticas (Fearnside 2003).

No estado do Pará estes perigos para a biodiversidade são claramente visíveis. As regióes da Rodovia Transamazônica (BR-230) e da volta grande do Rio Xingu, apresentam-se fortemente alteradas, em média, mais da metade de sua cobertura vegetal original foi convertida em diversos tipos de usos da terra, entre os quais agricultura familiar de ciclo curto, exploração madeireira, pastagens para pecuária bovina e culturas perenes, como cacau e pimenta do reino (Fearnside 2005; Ferreira et al. 2005; Salomão et al. 2007).

As variaçôes sazonais na regiāo amazônica são causadas principalmente pela precipitação, sendo esta a variável climatológica mais importante, dividindo o ano em dois períodos um mais e outro menos chuvoso (Moraes et al. 2005). Os estudos de diversidade temporal na Amazônia com invertebrados são escassos, porém importantes, pois nos trazem informaçóes necessárias sobre as variaçóes sazonais das espécies, demonstrando a época de ocorrência e permitindo um melhor conhecimento sobre o estado da fauna em determinado lugar.

Os insetos são considerados úteis para estudos de diversidade e avaliação de impactos no meio ambiente, devido serem fundamentais no funcionamento dos ecossistemas naturais, pois os mesmos atuam como predadores, fitófagos, parasitas, saprófagos e polinizadores (Rosenberg et al. 1986). Embora exista uma ampla diversidade de insetos na Amazônia, a grande maioria dos grupos ainda são pouco conhecidos (Henriques et al. 2007).

Devido aos lepidópteros serem uma das principais ordens de inseto, quanto à riqueza de espécies, sendo apropriados para estudos sobre a biodiversidade (Intachat e Woiwod 1999; Landau et al. 1999; Kitching et al. 2000; Camargo 2001). Assim, o estudo deste grupo pode contribuir para o conhecimento da maior parte das características de um determinado ambiente (Specht et al. 2005).
Deve se destacar, que entre os lepidópteros, os Arctiinae estão entre os mais importantes bioindicadores no monitoramento de ecossistemas (Hilty e Merenlender 2000) e, alguns estudos mais recentes vêm ressaltando esta importância, por serem bem conhecidos, relativamente fáceis de identificar, facilmente capturados e, como suas lagartas são fitófagas, respondem às alteraçôes na constituiçấo vegetal (Hilt e Fiedler, 2005; 2006; Brehm e Axmacher 2006; Brehm 2007; Hilt et al. 2007; Conner, 2009; Hawes et al. 2009; Teston et al. 2009; Teston e Delfina 2010). Na Região Neotropical são conhecidas 4.761 espécies de Arctiinae (Heppner 1991). Segundo Brown Jr e Freitas (1999), há uma estimativa de duas mil espécies para o Brasil. No Estado do Pará já foram descritas 485 espécies (Teston e Delfina 2010).

Este trabalho avaliou a fauna de Arctiinae noturnos, quanto a sua abundância, composição, diversidade e riqueza durante dois anos, entre agosto de 2007 a julho de 2009, em um fragmento de floresta na Amazônia Oriental em Altamira, Pará, a fim de se verificar se há variação sazonal nesta comunidade.

\section{MATERIAL E MÉTODOS}

O estudo foi desenvolvido na Base de Selva do $51^{\circ}$ Batalhão de Infantaria de Selva (51 $\left.{ }^{\circ} \mathrm{BIS}\right)$, localizado entre a latitude $03^{\circ} 11^{\prime} 55^{\prime}$ 'S e longitude $52^{\circ} 10^{\prime} 15^{\prime \prime W}$, com altitude média de 195 m, localizado no município de Altamira, Pará. As coletas foram mensais, durante uma noite a cada período de lua nova, devido a esta proporcionar noites mais escuras e não interferindo na captura, de agosto de $2007 \mathrm{a}$ julho de 2009, totalizando 24 amostragens. O fragmento de floresta possui uma área de 1.035 hectares de floresta primária de terra firme, apresentando-se de acordo com a caracterização feita por Salomão et al. (2007).

$\mathrm{Na}$ regiāo do estudo, o período mais chuvoso estende-se de dezembro a maio e o menos chuvoso de junho a novembro (Moraes et al. 2005). Conforme as informações obtidas junto a Estação de Meteorologia de Altamira, no $2^{\circ}$ Distrito de Meteorologia (DISME) do Instituto Nacional de Meteorologia (INMET), durante o período de amostragem, foi verificada uma temperatura média de $27.3^{\circ} \mathrm{C}$ e pluviosidade total de $5.249,5 \mathrm{~mm}$, sendo que no primeiro ano de coleta 27,2 ${ }^{\circ} \mathrm{C}$ e $2.399,7 \mathrm{~mm}$ e, no segundo $27,4{ }^{\circ} \mathrm{C}$ e $2.849,8 \mathrm{~mm}$ de temperatura média e pluviosidade, respectivamente.

Para a captura dos arctíneos foi utilizada armadilha luminosa EMATER/RS, modelo "Santa Rosa” (Kober 1982) adaptada, possuindo um tronco de cone plástico com maior diâmetro de $32 \mathrm{~cm}$ e menor de $16 \mathrm{~cm}$, ao qual estava acoplado um balde plástico com capacidade de 3,5 litros, onde foram colocados 2 litros de álcool 92。 GL. A armadilha estava equipada com uma lâmpada fluorescente ultravioleta F15 T8 
BLB, cuja luz possui um comprimento de onda que varia de 290 a 450 e pico ao redor de 340 nanômetros. A armadilha foi instalada no interior do fragmento florestal, cuja borda mais próxima estava a 700 metros, a uma altura de 2,5 metros do solo, acionada por bateria $12 \mathrm{~V}$, ao anoitecer $(18 \mathrm{~h})$ e desligada na manhã seguinte $(6 \mathrm{~h})$.

A triagem, identificação e contagem dos arctiíneos, foram realizadas no Laboratório Integrado de Biologia e Educação Ambiental (LIBEA) da Faculdade de Ciências Biológicas do Campus Universitário de Altamira da Universidade Federal do Pará (UFPA). O processo de identificação, o posicionamento sistemático adotado e a distribuição das espécies dentro dos gêneros e tribos seguem Teston et al. (2009) e Teston e Delfina (2010). Uma parte dos espécimes coletados foi preparada a seco, em alfinetes entomológicos, e a outra parte foi conservada em envelopes entomológicos. $\mathrm{O}$ material testemunha (coleçáo de referência) encontra-se depositado na Coleção Entomológica do Museu de Zoologia do Programa de Ciências Biológicas da Universidade Federal do Oeste do Pará (MZ-UFOPA), localizado no Campus Tapajós em Santarém, PA.

$\mathrm{Na}$ análise dos dados, calculou-se a constância das espécies em cada mês, classificando-as, segundo Bodenheimer referido por Silveira Neto et al. (1976), em: (1) constantes, onde as espécies estavam presentes em mais de $50 \%$ das coletas, (2) acessórias, presentes em $25 \%$ a $50 \%$ e (3) acidentais, presentes em menos de $25 \%$. Também foram medidos os seguintes os parâmetros: riqueza $(\mathrm{S})$, abundância $(\mathrm{N})$, índices de diversidade de Shannon ( $\left.\mathrm{H}^{\prime}\right)$ e de Brillouin $(\mathrm{H})$, índices de uniformidade de Shannon (E') e de Brillouin (E) e o índice de dominância de Berger-Parker (BP) (Magurran, 2004; Krebs, 1999). Todos os parâmetros foram calculados para os meses, ano e total. Os valores de $\mathrm{H}^{\prime}$, entre o $1^{\circ}$ e o $2^{\circ}$ ano de amostragem, foram comparados pelo teste " $\mathrm{t}$ " proposto por Hutcheson (1970). Foi avaliada também a similaridade entre os meses de coleta através do índice de Bray Curtis pelo método UPGMA, utilizando o software PAST versão 2.06 (Hammer et al. 2001). As estimativas de riqueza de espécies, para o período total de amostragem, foram realizadas com auxílio do programa "Estimates Richness Estimator" (Colwell 2009), empregando os procedimentos "Bootstrap", "Chao1", "Chao2", "Jackknife1", "Jackknife2" e "Michaelis-Mentem", utilizando 100 casualizaçôes com abundância de classes igual a 10 (Colwell e Coddington 1994). Também, foi construída a curva de rarefação de espécies utilizando o índice de "Mao Tau", com intervalo de confiança de 95\%.

\section{RESULTADOS E DISCUSSÃO}

Foram coletados 466 exemplares $(\mathrm{N})$ distribuídos em 78 espécies (S), das quais foram encontrados representantes para cinco das seis tribos ocorrentes na regiáo Neotropical
(Tabela 1), correspondendo a $16,08 \%$ do total conhecido para o Estado do Pará. Doze espécies são novos registros para o Estado: Amaxia reticulata (Rothschild, 1909), Cresera ilioides (Schaus, 1905), Demolis flavithorax (Rothschild, 1909), Himerarctia griseipennis (Rothschild, 1909), Hyperthaema coccinata, (Schaus, 1905), Idalus intermedia (Rothschild, 1909), Idalus vitrea (Cramer, 1780), Melese ocellata (Hampson, 1901), Neritos discophora (Hampson, 1901), Ormetica contraria (Walker, 1854), Trichromia polyxenoides (Rothschild, 1909) e Viviennea zonana (Schaus, 1905).

Em relação ao número mensal de espécies e exemplares capturados (Figura 1) nota-se uma tendência de se capturar mais exemplares e espécies durante os meses menos chuvosos (junho a novembro), para os períodos amostrados nos anos de 2008 e 2009, mas não para 2007. Com relação aos períodos 2008 e 2009, esta mesma tendência de capturar mais espécies e exemplares também foi observada por Teston e Delfina (2010), em um estudo em área alterada em Altamira.

Das 78 espécies capturadas, 70 (89,7\%) ocorreram com menos de 12 exemplares e destas, 44 estáo representadas por somente um exemplar ("singletons") e, quatro por dois exemplares ("doubletons"). Por outro lado, somente oito espécies ocorreram com 12 ou mais exemplares (10,3\%), destacando Virbia subapicalis (Walker, 1854) como a mais abundante (137 exemplares) (Tabela 1), ao contrário do que ocorreu na área alterada onde foram capturados somente três exemplares desta espécie (Teston e Delfina 2010). O fato de encontrar muitas espécies com poucos indivíduos é um padrão para comunidades de Arctiidae na regiâo Neotropical e já foi encontrado em alguns estudos efetuados no Brasil (Teston e Corseuil 2004; Ferro e Diniz 2007; Teston et al. 2009; Teston e Delfina 2010). Este fato anterior, também pode ser verificado pela constância das espécies (Tabela 1) onde, 67 espécies foram acidentais (85,9\%), oito acessórias $(10,3 \%)$ e apenas três constantes $(3,8 \%)$. Entre as constantes, Neritos discophora Hampson, 1901 foi capturada em 14 meses,

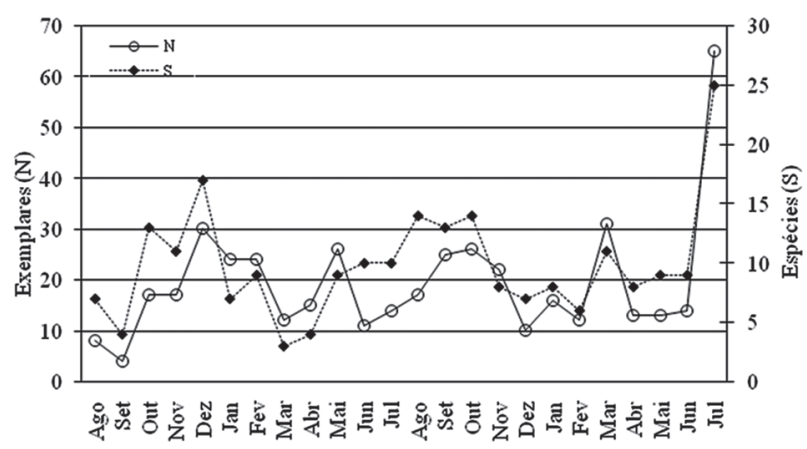

Figura 1 - Números de exemplares e espécies para Arctiinae capturados com armadilha luminosa em um fragmento florestal em Altamira, PA, Brasil, no período de agosto de 2007 a julho de 2009. 
Tabela 1 - Número de espécimes e constância ( $\mathrm{A}$ = Acidentais; $\mathrm{B}=$ Acessórias; e $\mathrm{C}=$ Constantes) para Arctiinae capturados com armadilha luminosa em um fragmento florestal em Altamira, PA, Brasil, no período de agosto de 2007 a julho de 2009.

\begin{tabular}{|c|c|c|c|c|c|c|c|c|c|c|c|c|c|c|c|c|c|c|c|c|c|c|c|c|}
\hline \multirow{2}{*}{ Tribos/espécies } & \multicolumn{4}{|c|}{2007} & \multicolumn{12}{|c|}{2008} & \multicolumn{7}{|c|}{2009} & \multirow{2}{*}{ 要 } \\
\hline & A S & 0 & $\mathrm{~N}$ & $\mathrm{D}$ & $\mathrm{J}$ & $\mathrm{F}$ & M & A & $M$ & $\mathrm{~J}$ & $\mathrm{~J}$ & A & $\mathrm{S}$ & 0 & $\mathrm{~N}$ & $\mathrm{D}$ & $\mathrm{J}$ & $\mathrm{F}$ & $\mathrm{M}$ & A & $\mathrm{M}$ & J & $\mathrm{J}$ & \\
\hline \multicolumn{25}{|l|}{ ARCTIINI } \\
\hline Paracles sp. ${ }^{A}$ & & & & & & & & & & & & & & & & 1 & & & & & & & 1 & 2 \\
\hline Virbia subapicalis (Walker, 1854) c & 21 & 3 & 7 & 12 & 15 & 15 & 7 & 11 & 11 & 2 & 4 & 2 & 6 & 5 & 8 & 4 & 1 & 1 & 10 & 2 & & 4 & 4 & 137 \\
\hline Virbia sp. $1^{\text {B }}$ & & & & & & & & & & & & 2 & 3 & & 3 & & 7 & & 6 & 1 & & & 6 & 28 \\
\hline Virbia sp. $2^{\mathrm{A}}$ & & & & & & & & & & & & & & 1 & & & & & & & & & & 1 \\
\hline \multicolumn{25}{|l|}{ CTENUCHINI } \\
\hline Aclytia heber (Cramer, 1780) A & & & & 1 & & & & & & & & & 2 & 2 & & & & & & & & 1 & & 6 \\
\hline Aethria carnicauda (Butler, 1876) ${ }^{\text {A }}$ & & & & & & & & 1 & & & & & & & & & & & & & & & & 1 \\
\hline Correbia lycoides (Walker, 1854) A & & 1 & & & & & & & & & & & & & & & & & & & & & & 1 \\
\hline Correbidia notata (Butler, 1878) ${ }^{\mathrm{A}}$ & & & & & & & & & & & & & & & & & & & & & & & 1 & 1 \\
\hline Correbidia sp. ${ }^{\mathrm{A}}$ & & & & & & & & & & 1 & & & & & & & & & & & & & & 1 \\
\hline Delphyre discalis (Druce, 1905) ${ }^{\mathrm{A}}$ & & & & 2 & & & & & & & & & & & & & & & & & & & & 2 \\
\hline Delphyre parcipuncta (Hampson, 1914) & & 1 & & 1 & & & & & & & & 1 & 1 & & & & 1 & & & 1 & & & 1 & 7 \\
\hline Ecdemus hypoleucus (Herrich-Schäffer, 1855) & & & & & & & & & & & & & 1 & & & & & & & & & & & 1 \\
\hline Epidesma aurimacula (Schaus, 1905) A & & & & & & & & & & & & & 1 & & & & & & & & & & & 1 \\
\hline Epidesma ursula (Stoll, 1781) A & & & & & & & & & & & & & & & & & & & & & & & 1 & 1 \\
\hline Epidesma sp. $1^{\mathrm{A}}$ & & & & & & & & & & & & & & & & & 1 & & & & & & & 1 \\
\hline Epidesma sp. $2^{\text {A }}$ & & & & & & & & & & & & 1 & & & & & & & & & & & & 1 \\
\hline Episcepsis frances (Dyar, 1910) A & & & & & & & & & & & & 1 & & & & & & & & & & & & 1 \\
\hline Episcepsis sp. ${ }^{\text {A }}$ & & & & & & 1 & & & & & & & & & & & & & & & & & & 1 \\
\hline Eucereon balium (Hampson, 1898) A & & & & & & & & & & & 1 & 1 & & & & & & & & & & & & 2 \\
\hline Eucereon obscurum (Möschler, 1872) A & & & 1 & & & & & & 1 & & & & & & & & & & & & & & & 2 \\
\hline Eucereon sp. ${ }^{A}$ & & & & 1 & & & & & & & & & & & & & & & & & & & & 1 \\
\hline Napata leucotela (Butler, 1876) A & 1 & & & & & & & & & & & & & & & & & & & & & & & 1 \\
\hline Ptychotricos zeus (Schaus, 1894) ${ }^{\text {A }}$ & & & & & & & & & & 1 & & & & & & & & & & & & & & 1 \\
\hline \multicolumn{25}{|l|}{ EUCHROMIINI } \\
\hline Calonotos triplaga (Hampson, 1909) A & & & & & & & & & & & & & & & & & 1 & & & & & & & 1 \\
\hline Cosmosoma metallescens (Ménétries, 1857) & & & & 1 & & & & & & & & & 1 & & & & 1 & & & & 1 & & & 4 \\
\hline Dycladia lucetius (Stoll, 1781) A & & & & & & 1 & & & & & & & & & & & & & & & 1 & & 1 & 3 \\
\hline Histioea cepheus (Cramer, 1780) A & & & & 1 & & & & & & & & & & & & & & & & & & & & 1 \\
\hline Isanthrene profusa (Hampson, 1898) ${ }^{\text {в }}$ & & 1 & & 1 & & 1 & & & 1 & 1 & 1 & & 2 & 2 & 1 & & & 1 & & & & & & 12 \\
\hline Leucotmemis torrida (Walker, 1854) ${ }^{\text {B }}$ & & 1 & & & 3 & 1 & & 1 & & 1 & 1 & & & & 4 & & & & 2 & & 2 & & 3 & 19 \\
\hline Macrocneme sp. ${ }^{A}$ & & & & & & & & & & & & & 1 & & & & & & & & & & & 1 \\
\hline Poecilosoma eone (Hübner, 1827) A & & & 1 & & & & & & & & & & & & & & & & & & & & & 1 \\
\hline Saurita tipulina (Hübner, 1827) A & & & 1 & & & & & & & & & & 1 & & & & & & 1 & & & & & 3 \\
\hline \multicolumn{25}{|l|}{ PERICOPINI } \\
\hline Calodesma collaris (Drury, 1782) A & & & & 1 & 1 & & & & & & & 1 & & & & & & & & & & & & 3 \\
\hline Dysschema tricolor (Sulzer, 1776) A & & & & & & & & & & & & & & & & & & & & & & 1 & & 1 \\
\hline Hyalurga fenestra (Linnaeus, 1788) A & & & & 1 & & & & & & & & & & & & & & & & & & & & 1 \\
\hline Hyalurga leucophlebia (Hering, 1925) ${ }^{\mathrm{B}}$ & & 1 & & & & & & & & & & & & 1 & & & & 1 & 1 & 1 & & 1 & 2 & 8 \\
\hline Hyalurga mysis (Erichson, 1848) A & 1 & & & & & & & & & 1 & & & & & & & & 1 & 1 & & & & & 4 \\
\hline
\end{tabular}


Tabela 1 - Continuaçao.

\begin{tabular}{|c|c|c|c|c|c|c|c|c|c|c|c|c|c|c|c|c|c|c|c|c|c|c|c|c|}
\hline \multirow{2}{*}{ Tribos/espécies } & \multicolumn{4}{|c|}{2007} & \multicolumn{12}{|c|}{2008} & \multicolumn{7}{|c|}{2009} & \multirow{2}{*}{ 要 } \\
\hline & A S & 0 & $\mathrm{~N}$ & $\mathrm{D}$ & $\mathrm{J}$ & $\mathrm{F}$ & $\mathrm{M}$ & $\mathrm{A}$ & $\mathrm{M}$ & $\mathrm{J}$ & $\mathrm{J}$ & A & $S$ & 0 & $\mathrm{~N}$ & $\mathrm{D}$ & $\mathrm{J}$ & $\mathrm{F}$ & $\mathrm{M}$ & A & $\mathrm{M}$ & $\mathrm{J}$ & $\mathrm{J}$ & \\
\hline \multicolumn{25}{|l|}{ PHAEGOPTERINI } \\
\hline Agaraea minuta (Schaus, 1892) ${ }^{\mathrm{A}}$ & & & & 1 & & & & & & & & & & & & & & & & & & & & 1 \\
\hline Agaraea semivitrea (Rothschild, 1909) A & & & 1 & & & & & & & & & & & & & & & & & & & & & 1 \\
\hline Amaxia consistens (Schaus, 1905) A & 1 & & & & 1 & & & & & & 1 & & & & & & & & 1 & & & & 3 & 7 \\
\hline Amaxia lepida (Schaus, 1912) ${ }^{\text {A }}$ & & & 1 & & & & & & & & & & & & & & & & & & & & & 1 \\
\hline Amaxia reticulata (Rothschild, 1909) A & & & & & & & & & & & & 1 & & 1 & & 1 & & & & & & & 1 & 4 \\
\hline Amaxia sp. ${ }^{A}$ & & & & & & & & & & & & & & & & & & & & & & & 1 & 1 \\
\hline Ammalo helops (Cramer, 1775) ${ }^{A}$ & & & & & & & & & 1 & & & & & & & & & & & & & & & 1 \\
\hline Cresera affinis (Rothschild, 1909) A & & & & & & & & & & & & & & & & & & & & & & & 1 & 1 \\
\hline Cresera ilioides (Schaus, 1905) A & & & & & & & & & 1 & & & & & & & & & & & & & & & 1 \\
\hline Demolis flavithorax (Rothschild, 1909) ${ }^{\text {B }}$ & & 1 & & 1 & 1 & 1 & & & & & & 1 & 1 & & & & & & & & & & 2 & 8 \\
\hline Euplesia sphingidea (Perty, 1833) A & & & & & & & & & & & & & & 1 & & & & & & & & & & 1 \\
\hline Eupseudosoma larissa (Druce, 1890) A & & & & & & & & & & & & 1 & & & & & & & & & & & & 1 \\
\hline Halysidota sannionis (Rothschild, 1909) c & 1 & 1 & 1 & 1 & 2 & 2 & 4 & 2 & 6 & & 2 & & 4 & 6 & & 1 & 3 & 4 & 3 & 2 & 2 & 1 & 5 & 53 \\
\hline Halysidota sp. ${ }^{\text {A }}$ & & & & & & & & & & & & & & & & & & & & & 1 & & & 1 \\
\hline Himerarctia griseipennis (Rothschild, 1909)A & & & & & & & & & & & & & 1 & 1 & & 1 & & & & & & & & 3 \\
\hline Hyperandra novata (Dognin, 1924) A & & & & 1 & & & & & & & 1 & & & 1 & & & & & & & & & & 3 \\
\hline Hyperthaema coccinata, (Schaus, 1905) A & & & & & 1 & & & & & & & & & & & & & & & & & & & 1 \\
\hline Idalus aleteria (Schaus, 1905) ${ }^{A}$ & & 1 & & & & & & & & 1 & & & & & & & & & & & & & 1 & 3 \\
\hline Idalus critheis (Druce, 1884) ${ }^{\mathrm{A}}$ & 1 & & & & & & & & & & & & & & 1 & & & & & & & & 1 & 3 \\
\hline Idalus admirabilis (Cramer, 1777) ${ }^{\mathrm{A}}$ & & & & & & & & & & & & & & & & & & & & & & 1 & & 1 \\
\hline Idalus intermedia (Rothschild, 1909) A & & & & & & & & & 1 & & & & & & & & & & & & & & & 1 \\
\hline Idalus vitrea (Cramer, 1780) ${ }^{\text {В }}$ & & & 1 & 1 & & & & & 1 & & 1 & & & & 2 & & & & 1 & & & & 5 & 12 \\
\hline Lepidokirbya vittipes (Walker, 1855) A & & & 1 & & & & & & & 1 & & & & 1 & & 1 & & & & & & & & 4 \\
\hline Leucanopsis sp. $1^{\text {A }}$ & & & & & & & & & & & & 1 & & & & & & & & & 2 & 1 & 2 & 6 \\
\hline Leucanopsis sp. $2^{\mathrm{A}}$ & & & & & & & & & & & & & & 1 & & & & & & & & & & 1 \\
\hline Lophocampa sp. ${ }^{\text {A }}$ & 1 & & & & & & & & & & & 1 & & & & & & & & & 1 & & 1 & 4 \\
\hline Melese drucei (Rothschild, 1909) A & & & & & & & & & & & & & & & & & & & & & & & 1 & 1 \\
\hline Melese incertus (Walker, 1855) A & & & & & & & & & & & & & & & & & & & & & 1 & & & 1 \\
\hline Melese ocellata (Hampson, 1901) B & & & 1 & 2 & & & & & & 1 & 1 & & & 2 & & & & & & 1 & & & & 8 \\
\hline Neonerita dorsipuncta (Hampson, 1901) A & & & & & & & & & & & & & & & & & & & & 1 & & & & 1 \\
\hline Neritos discophora (Hampson, 1901) ${ }^{c}$ & 1 & & 1 & 1 & & 1 & 1 & & & & 1 & & & 1 & 2 & & 1 & 4 & 4 & 4 & & 2 & 3 & 27 \\
\hline Ormetica contraria (Walker, 1854) A & & 1 & & & & & & & & & & & & & & & & & & & & & & 1 \\
\hline Ormetica sypilus (Cramer, 1777) ${ }^{\text {A }}$ & & & & & & & & & & 1 & & & & & & & & & & & & & & 1 \\
\hline Pareuchaetes aurata (Butler, 1875) A & 1 & & & & & & & & & & & 2 & & & & & & & & & 2 & 2 & 16 & 23 \\
\hline Psycophasma erosa (Herrich-Schäffer, 1858)A & & 1 & & & & & & & & & & 1 & & & & 1 & & & & & & & & 3 \\
\hline Rhipha strigosa (Walker, 1854) A & & & & & & & & & & & & & & & & & & & & & & & 1 & 1 \\
\hline Trichromia cyclopera (Hampson, 1905) ${ }^{A}$ & & & & & & 1 & & & & & & & & & & & & & & & & & & 1 \\
\hline Trichromia polyxenoides (Rothschild,1909) A & & & & & & & & & & & & & & & & & & & 1 & & & & & 1 \\
\hline Viviennea moma (Schaus, 1905) A & 1 & 1 & & & & & & & & & & & & & & & & & & & & & 1 & 3 \\
\hline Viviennea superba (Druce, 1883) A & & 3 & & & & & & & 3 & & & & & & & & & & & & & & & 6 \\
\hline Viviennea zonana (Schaus, 1905) A & & & & & & & & & & & & & & & 1 & & & & & & & & & 1 \\
\hline SOMA & 84 & 17 & 17 & 30 & 24 & 24 & 12 & 15 & 26 & 11 & 14 & 17 & 25 & 26 & 22 & 10 & 16 & 12 & 31 & 13 & 13 & 14 & 65 & 466 \\
\hline
\end{tabular}


Halysidota sannionis (Rothschild, 1909) em 20 meses e Virbia subapicalis (Walker, 1854) em 23 meses. Destas três, somente Virbia subapicalis ocorreu na área alterada (Teston e Delfina 2010) em somente dois meses.

$\mathrm{Na}$ Tabela 2, encontramos os valores para os parâmetros riqueza de espécies $(\mathrm{S})$, número de espécimes $(\mathrm{N})$, índices de diversidade de Shannon ( $\left.\mathrm{H}^{\prime}\right)$ e de Brillouin $(\mathrm{H})$, uniformidade de Shannon (E') e Brillouin (E) e dominância de Berger-Parker (BP) para os meses, ano e período total.

Entre os meses, a maior riqueza de espécies foi encontrada em julho de 2009 (25 espécies). Este período também apresentou os maiores índices de diversidade de Shannon $\left(\mathrm{H}^{\prime}=2,78\right)$ e Brillouin $(\mathrm{H}=2,34)$ (Tabela 2).

Com relação à uniformidade máxima, estas foram encontradas para Shannon no mês de setembro de 2007 $\left(E^{\prime}=1,00\right)$ e, para Brillouin nos meses de agosto e setembro de 2007, junho e agosto de 2008 e maio de $2009(E=1,00)$ (Tabela 2).

A dominância de Berger-Parker (BP) foi mais alta no mês de abril de $2008(\mathrm{BP}=0,733)$ e mais baixa em agosto do mesmo ano (Tabela 2). Devido forte dominância que ocorreu no mês de abril de 2008 os parâmetros diversidade e uniformidade foram os mais baixos em todos os índices calculados (Shannon e Brillouin) e isto é característico porque a diversidade e a uniformidade são inversamente proporcionais a dominância.

Quando comparamos o primeiro ano com o segundo (Tabela 2), observamos que todos os parâmetros avaliados foram superiores no segundo ano, riqueza, abundância, índices de diversidade e uniformidade de Shannon e Brillouin são maiores numericamente e dominância de BergerParker mais baixa. O índice de diversidade de Shannon foi significativamente diferente pelo teste " $\mathrm{t}$ " entre o $1^{\circ}$ ano (2007-2008) e o $2^{\circ}$ ano (2008-2009). O teste " $\mathrm{t}$ " se faz necessário, pois este índice assume que a amostra é uma parte da populaçáo então, para podermos compará-lo entre os períodos devemos realizar o teste de significância estatística. Isto não é necessário para o índice de Brillouin, devido a ele admitir que a amostra é a própria população então o valor em si já é diferente.

Para todo o período de amostragem (Tabela 2), foram encontrados os seguintes valores para a diversidade: $\mathrm{H}^{\prime}=3,08 \mathrm{e}$ $\mathrm{H}=2,86$; uniformidade: $\mathrm{E}^{\prime}=0,708$ e $\mathrm{E}=0,705$; e dominância: $\mathrm{BP}=0,294$.

$\mathrm{Na}$ analise de similaridade (Figura 2), segundo índice de Bray Curtis, não ocorreu um separação evidente entre os meses do período menos e mais chuvosos. Na primeira divisão o mês de setembro de 2007 (menos chuvoso) foi separado dos demais. Na segunda divisão os meses de maio de 2009 (mais chuvoso), julho de 2009 e agosto de 2008 (menos chuvosos)
Tabela 2 - Valores de riqueza (S) e abundância (N) de espécies, índices de diversidade de Shannon $\left(\mathrm{H}^{\prime}\right)^{*}$ e de Brillouin $(\mathrm{H})^{*}$, índices de uniformidade de Shannon (E') e de Brillouin (E) e de dominância de Beger-Parker (BP) para Arctiinae capturados com armadilha luminosa em um fragmento florestal em Altamira, PA, Brasil, no período de agosto de 2007 a julho de 2009.

\begin{tabular}{|c|c|c|c|c|c|c|c|c|}
\hline ANO & MESES & S & $\mathrm{N}$ & $\mathrm{H}^{\prime}$ & $E^{\prime}$ & $\mathrm{H}$ & $E$ & $\mathrm{BP}$ \\
\hline \multirow{5}{*}{ 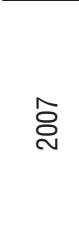 } & AGO & 7 & 8 & 1,91 & 0,980 & 1,24 & 1,00 & 0,250 \\
\hline & SET & 4 & 4 & 1,39 & 1,00 & 0,79 & 1,00 & 0,250 \\
\hline & OUT & 13 & 17 & 2,45 & 0,953 & 1,76 & 0,974 & 0,176 \\
\hline & NOV & 11 & 17 & 2,03 & 0,847 & 1,47 & 0,851 & 0,412 \\
\hline & DEZ & 17 & 30 & 2,31 & 0,812 & 1,78 & 0,812 & 0,400 \\
\hline \multirow{7}{*}{ 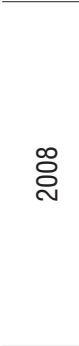 } & JAN & 7 & 24 & 1,29 & 0,663 & 1,02 & 0,641 & 0,625 \\
\hline & FEV & 9 & 24 & 1,43 & 0,650 & 1,09 & 0,624 & 0,625 \\
\hline & MAR & 3 & 12 & 0,89 & 0,808 & 0,69 & 0,792 & 0,583 \\
\hline & $A B R$ & 4 & 15 & 0,86 & 0,618 & 0,65 & 0,586 & 0,733 \\
\hline & MAl & 9 & 26 & 1,70 & 0,775 & 1,36 & 0,765 & 0,423 \\
\hline & JUN & 10 & 11 & 2,27 & 0,987 & 1,53 & ,00 & 0,182 \\
\hline & JUL & 10 & 14 & 2,14 & 0,931 & 1,52 & 0,951 & 0,286 \\
\hline \multicolumn{2}{|c|}{$1^{\circ}$ Ano (2007-2008) } & 49 & 202 & $2,55^{t}$ & 0,656 & 2,27 & 0,646 & 0,446 \\
\hline \multirow{5}{*}{ 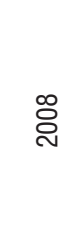 } & AGO & 14 & 17 & 2,59 & 0,981 & 1,85 & 1,00 & 0,118 \\
\hline & SET & 13 & 25 & 2,32 & 0,906 & 1,80 & 0,907 & 0,240 \\
\hline & OUT & 14 & 26 & 2,38 & 0,900 & 1,84 & 0,903 & 0,231 \\
\hline & NOV & 8 & 22 & 1,81 & 0,869 & 1,43 & 0,867 & 0,364 \\
\hline & DEZ & 7 & 10 & 1,75 & 0,898 & 1,19 & 0,916 & 0,400 \\
\hline \multirow{7}{*}{ 옹 } & JAN & 8 & 16 & 1,72 & 0,825 & 1,27 & 0,810 & 0,438 \\
\hline & FEV & 6 & 12 & 1,56 & 0,871 & 1,14 & 0,861 & 0,333 \\
\hline & MAR & 11 & 31 & 2,01 & 0,840 & 1,64 & 0,838 & 0,323 \\
\hline & $A B R$ & 8 & 13 & 1,93 & 0,926 & 1,38 & 0,942 & 0,308 \\
\hline & MAI & 9 & 13 & 2,14 & 0,973 & 1,52 & 1,00 & 0,154 \\
\hline & JUN & 9 & 14 & 2,04 & 0,931 & 1,47 & 0,949 & 0,286 \\
\hline & JUL & 25 & 65 & 2,78 & 0,863 & 2,34 & 0,865 & 0,246 \\
\hline \multicolumn{2}{|c|}{$2^{\circ}$ Ano(2008-2009) } & 55 & 264 & $3,13^{t}$ & 0,780 & 2,86 & 0,781 & 0,178 \\
\hline \multicolumn{2}{|l|}{ TOTAL } & 78 & 466 & 3,08 & 0,708 & 2,86 & 0,705 & 0,294 \\
\hline
\end{tabular}

* (Logaritmo Natural)

$\mathrm{t}=$ significativamente diferente ao nível de $5 \%$ pelo teste "t"

foram separados dos outros meses. Na terceira divisão os meses de agosto e outubro de 2007 e junho de 2008 (menos chuvosos) foram separados dos demais. Na quarta divisão os meses de janeiro, fevereiro e abril de 2009 (mais chuvosos) foram separados dos outros meses. Na quinta divisão os meses de dezembro de 2008 (mais chuvoso) e junho de 2009 (menos chuvoso) foram separados dos demais. $\mathrm{Na}$ sexta divisão os meses de setembro e outubro de 2008 (menos chuvosos) foram separados dos outros meses. Na sétima divisão os meses novembro de 2007 (menos chuvoso), março (mais chuvoso) e julho de 2008 (menos chuvoso) foram separados dos demais. 


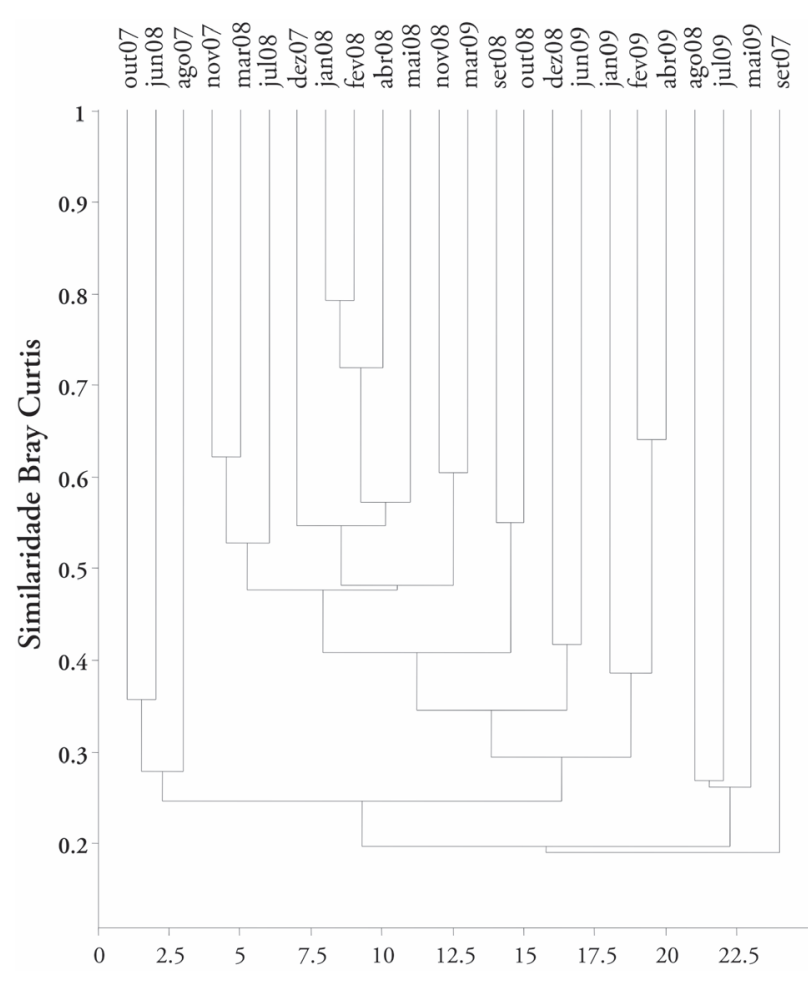

Figura 2 - Análise de agrupamento (UPGMA) baseado no índice de Bray Curtis para Arctiinae capturados com armadilha luminosa em um fragmento florestal em Altamira, PA, Brasil, no período de agosto de 2007 a julho de 2009.

$\mathrm{Na}$ oitava divisão os meses de novembro de 2008 (menos chuvoso) e março de 2009 (mais chuvoso) foram separados dos outros meses. Esse agrupamento resultante une somente meses de período mais chuvoso (dezembro de 2007, janeiro, fevereiro, abril e maio de 2008), destacando que os meses de janeiro e fevereiro apresentaram a maior similaridade $(79,2 \%)$.

As estimativas de riqueza de espécies (Tabela 3) revelam que podemos encontrar entre 21,8\% ("Bootstrap") e 324,4\% ("Chao2") a mais de espécies. Esta forte porcentagem nas estimativas é devido ao elevado número de "Singletons" e "Doubletons" e demonstra que devemos encontrar muito mais espécies através da ampliação do período de amostragem e, ainda, indica que a riqueza de Arctiinae na floresta Amazônica é muito mais alta principalmente quando comparada com a estimativa máxima para área alterada que foi cinco vezes menor (Teston e Delfina 2010). Destacamos também, que em um estudo sobre diversidade de lepidópteros em florestas Hawes et al. (2009) também encontraram valores elevados nas estimativas de riqueza para o grupo em questáo.

A curva de rarefação de espécies (Figura 3) corrobora com o que foi apresentado pelos estimadores de riqueza, pois ela não apresenta uma tendência assíntota, mostrando que são necessárias mais amostras.
Tabela 3 - Estimativas para Arctiinae capturados com armadilha luminosa em um fragmento florestal em Altamira, PA, Brasil, no período de agosto de 2007 a julho de 2009. Número de amostras, espécies, números de espécies representadas por apenas um ("Singletons"), e dois exemplares ("Doubletons") e riqueza de espécies, segundo vários estimadores.

\begin{tabular}{lc}
\hline & Valores \\
\hline Amostras & 24 \\
Espécies & 78 \\
"Singletons" & 44 \\
"Doubletons" & 4 \\
"Bootstrap" & 95 \\
"Chao 1" & 320 \\
"Chao 2" & 331 \\
"Jackknife 1" & 121 \\
"Jackknife 2" & 159 \\
"Michaelis-Mentem" & 117 \\
\hline
\end{tabular}

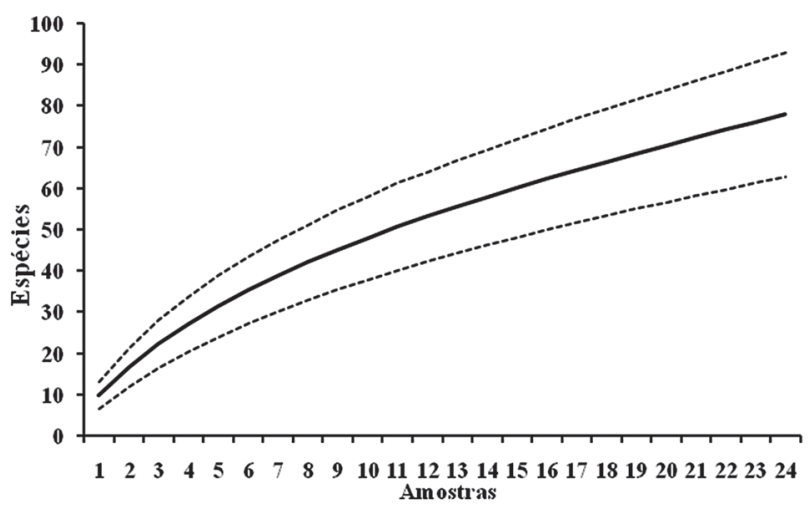

Figura 3 - Curva de rarefação de espécies de Arctiinae (Lepidoptera, Arctiidae) capturados com armadilha luminosa em um fragmento florestal em Altamira, Amazônia Oriental, Pará, Brasil, no período de agosto de 2007 a julho de 2009 , em relação ao esforço amostral, com os respectivos intervalos de confiança $95 \%$.

\section{CONCLUSÕES}

Virbia subapicalis foi a espécie mais abundante e freqüente. Destaca-se que $89,7 \%$ das espécies ocorreram com freqüências muito baixas, com menos de 12 exemplares, fato já observado para Arctiinae em outros locais no Brasil e também em uma área alterada no mesmo município. Julho de 2009 foi o mês de maior riqueza e abundância, isto deve ser devido a pouca variação pluviométrica, que poderia afetar a captura e o desenvolvimento biológico das espécies, este fato também já foi observado no estudo sobre Arctiinae na área alterada no município.

O período relativo aos anos 2008-2009 ( $2^{\circ}$ ano) foi superior em todos os parâmetros avaliados. Já, em relaçâo aos meses, julho de 2009 foi o que obteve melhores valores para a diversidade de Shannon e Brillouin. No quesito uniformidade, 
a de Shannon foi máxima em um único mês (setembro de 2007) já, para Brillouin em cinco (agosto e setembro de 2007, junho e agosto de 2008 e maio de 2009), excetuando maio de 2009 todos os demais meses são durante o período menos chuvoso. Com relação a dominância de Berger-Parker, o melhor valor (o mais baixo) foi encontrado em agosto de 2008, também durante o período menos chuvoso.

A analise de agrupamento náo demonstrou haver uma diferença na similaridade entre os períodos menos e mais chuvoso, porém, houve um agrupamento envolvendo cinco meses durante o período mais chuvoso de 2007 e 2008. E, a maior similaridade $(79,2 \%)$ foi encontrada entre os meses de janeiro e fevereiro de 2008.

Com relação às estimativas de riqueza, apuramos uma amplitude de variaçáo muito alta entre $21,8 \%$ e $324,4 \%$ o que permite concluir que mais amostras ao longo do tempo devam ser realizadas e que a riqueza de Arctiinae é muito alta em florestas na Amazônia.

Registram-se mais doze espécies para o Estado, ampliando desta forma o conhecimento sobre as espécies ocorrentes no Pará.

\section{AGRADECIMENTOS}

Aos Comandantes do $51^{\circ}$ Batalhão de Infantaria de Selva, que possibilitaram o acesso a Base de Selva e pelo apoio durante todo período da pesquisa. A Patrícia Lazamé Conceiçáo, da Estação de Meteorologia de Altamira (2。 DISME - INMET), pelas informações meteorológicas. A Fundação de Amparo à Pesquisas do Estado do Pará (FAPESPA) por conceder auxílio financeiro para pesquisas com Arctiinae através do Edital Universal 2008 (Termo de Outorga e Aceitação de Auxílio n. ${ }^{\circ}$ 138/2008), o qual permitiu a conclusão deste trabalho. Ao Conselho Nacional de Desenvolvimento Científico e Tecnológico (CNPq) pela cota de bolsa PIBIC/CNPq 2007/2008 e a Universidade Federal do Pará pela cota de bolsa PIBIC/UFPA 2008/2009.

\section{BIBLIOGRAFIA CITADA}

Brehm, G. 2007. A Contrasting patterns of vertical stratification in two moths families in Costa Rican lowland rain forest. Basic and Applied Ecology, 8: 44-54.

Brehm, G.; Axmacher, J.C. 2006. A Comparison of Manual and Automatic Moth Sampling Methods (Lepidoptera: Arctiidae, Geometridae) in a Rain Forest in Costa Rica. Environmental Entomology, 35: 757-764.

Brown Jr., K.S.; Freitas, A.V.L. 1999. Lepidoptera, p. 225-243. In: Brandão, C.R.F.; Cancello, E.M. (Eds). Biodiversity of the State of São Paulo: synthesis of knowledge at the end of the twentieth century. Volume 5. Invertebrates. FAPESP, São Paulo (in Portuguese).

Camargo, A.J.A. de. 2001. Insect Diversity in Cultivated Areas and Legal Reserves: Considerations and Recommendations. EMBRAPA
Distrito Federal, Planaltina. 27 pp. (Boletim de Pesquisa e Desenvolvimento, 1) (in Portuguese, with abstract in English).

Colwell, R.K. 2009. EstimateS: Statistical estimation of species richness and shared species from samples. Version 8.2.0. User's Guide and application. (http://viceroy.eeb.uconn.edu/EstimateS). Acesso: $14 / 10 / 2010$.

Colwell, R.K.; Coddington, J.A. 1994. Estimating terrestrial biodiversity through extrapolation. Philosophical Transaction of the Royal Society of London, Series B, 345: 101-118.

Conner, W.E. 2009. Tiger Moths and Woolly Bears: Behavior, ecology, and evolution of the Arctiidae. Oxford University Press, New York. 303 pp.

Fearnside, P.M. 2003. The Amazon rainforest in global change. INPA, Manaus. 134 pp (in Portuguese).

Fearnside, P.M. 2005. Deforestation in Brazilian Amazonia: history, rates and consequences. Megadiversidade, 1: 113-123 (in Portuguese, with abstract in English).

Ferreira, L.V.; Venticinque, E.; Almeida, S. 2005. Deforestation in the Amazon and the importance of protected areas. Estudos Avançados, 19: 157-166 (in Portuguese, with abstract in English).

Ferro, V.G.; Diniz, I.R. 2007. Composition of the Arctiidae species (Insecta, Lepidoptera) in Cerrado areas. Revista Brasileira de Zoologia, 24: 635-646 (in Portuguese, with abstract in English).

Hammer, Ø; Harper, D.A.T.; Ryan, P.D. 2001. PAST: Paleontological Statistics software package for education and data analysis. Palaeontologia Electronica, 4: 1-9.

Hawes, J.; Motta, C. da S.; Overal, W.L.; Barlow, J.; Gardner, T.A.; Peres, C.A. 2009. Diversity and composition of amazonian moths in primary, secondary and plantation forest. Journal of Tropical Ecology, 25: 281-300.

Henriques, A.L.; Rafael, J.A.; Ale-Rocha, R.; Xavier-Filho, F.F.; Baccaro, F.B.; Godoi, F.S.P. 2007. Insects and other terrestrial arthropods, p. 57-68. In. Rapp Py-Daniel, L.; deus, C.P.; Henriques, A.L.; Pimpão, D.M.; Ribeiro, O.M. (Orgs.). Biodiversity in the Middle Madeira: The scientific basis for conservation proposals. INPA, Manaus (in Portuguese).

Heppner, J.B. 1991. Faunal regions and the diversity of Lepidoptera. Tropical Lepidoptera, 2(suppl. 1): 1-85.

Hilt, N.; Brehm, G.; Fiedler, K. 2007. Temporal dynamics of rich moth ensembles in the montane forest zone in southern Ecuador. Biotropica, 39: 94-104.

Hilt, N.; Fiedler, K. 2005. Diversity and composition of Arctiidae moth ensembles along a successional gradient in the Ecuadorian Andes. Diversity and Distributions, 11: 387-398.

Hilt, N.; Fiedler, K. 2006. Arctiidae moth ensembles along a successional gradient in the Ecuadorian montane rain forest zone: how different are subfamilies and tribes? Journal of Biogeography, 33: 108-120.

Hilty, J.; Merenlender, A. 2000. Faunal indicator taxa selection for monitoring ecossystem health. Biological Conservation, 92: 185-197.

Hutcheson, K. 1970. A test for comparing diversities based on Shannon formula. Journal of Theoretical Biology, 29: 151-154. 
Intachat, J.; Woiwod, I.P. 1999. Trap design for monitoring moth biodiversity in tropical rainforests. Bulletin of Entomological Research, 89: 153-163.

Kitching, R.L.; Orr, A.G.; Thalib, L.; Mitchell, H.; Hopkins, M. S.; Graham, A.W. 2000. Moths assemblages as indicators of environmental quality in remnants of upland Australian rain forest. Journal of Applied Ecology, 37(2): 284-297.

Kober, E.A.M. 1982. Light trap technical information. EMATER/ RS, Porto Alegre. 24 pp (in Portuguese).

Krebs, C.J. 1999. Ecological Methodology. 2a ed. Benjamin/ Cummings, New York. 620 pp.

Landau, B.; Prowell, D.; Carlton, C.E. 1999. Intensive versus longterm sampling to assess lepidopteran diversity in southern mixed mesophytic forest. Annals of the Entomological Society of America, 92(3): 435-441.

Magurran, A.E. 2004. Measuring Biological Diversity. Blackwell Publishing, Oxford. 260 pp.

Moraes, B.C. de; Costa, J.M.N. da; Costa, A.L. da; Costa, M.H. 2005. Spatial and temporal variation of precipitation in the State of Pará. Acta Amazonica, 35: 207-214 (in Portuguese, with abstract in English).

Overal, W.L. 2001. The weight of invertebrates in the balance of biological conservation in the Amazon, p. 50-59. In: Capobianco, J.P.R. et al. (Orgs.). Biodiversity in the Brazilian Amazon. Estação Liberdade/Instituto Socioambiental, São Paulo (in Portuguese).

Primack, R.B.; Rodrigues, E. 2001. Conservation Biology. Ed. Planta, Londrina. 328 pp (in Portuguese).

Rosenberg, D.M.; Danks, H.V.; Lehmkuhl, D.M. 1986. Importance of insects in environmental impact assessment. Environmental Management, 10: 773-783.
Salomão, R. de P.; Vieira, I.C.G.; Suemitsu, C.; Rosa, N. de A.; Almeida, S.S. de ; Amaral, D.D. do; Menezes, M.P.M. de. 2007. The forests of Belo Monte on the great curve of the Xingu River, Eastern Amazon. Boletim Museu Paraense Emílio Goeldi. Ciências Naturais, 2: 57-153 (in Portuguese, with abstract in English).

Silva, J.M.C. da; Garda, A.A. 2011. Biogeographic patterns and processes in the Amazon, p. 189-197. In: Carvalho, C.J.B. de; Almeida, E.A.B. (Orgs.). Biogeography of South America: patterns and processes. Roca, São Paulo (in Portuguese).

Silveira Neto, S.; Nakano, O.; Barbin, D.; Nova, N.A.V. 1976. Manual of Insect Ecology. Agronômica Ceres, Piracicaba. 419 pp (in Portuguese).

Specht, A; Teston, J.A.; Di Mare, R.A.; Corseuil, E. 2005. Owlet moths (Lepidoptera, Noctuidae) collected in four protected areas located in Rio Grande do Sul State, Brazil. Revista Brasileira de Entomologia, 49: 130-140 (in Portuguese, with abstract in English).

Teston, J.A.; Corseuil, E. 2004. Diversity of Arctiinae (Lepidoptera, Arctiidae) captured by light traps in six communities in Rio Grande do Sul State, Brazil. Revista Brasileira de Entomologia, 48: 77-90 (in Portuguese, with abstract in English).

Teston, J.A.; Delfina, M.C. 2010. Diversity of Arctiinae (Lepidoptera, Arctiidae) in disturbed area at Altamira, Eastern Amazon, Pará, Brazil. Acta Amazonica, 40: 387-396 (in Portuguese, with abstract in English).

Teston, J.A.; Silveira, A.P.; Corseuil, E. 2009. Abundance, Composition and Diversity of Arctiinae (Lepidoptera, Arctiidae) in a Atlantic forest fragment in Iraí, RS, Brazil. Revista Brasileira de Zoociencias, 11: 65-72 (in Portuguese, with abstract in English).

Recebido em 17/11/2010

Aceito em 13/04/2011 
